- Develop and maintain a representative workforce.

- Hear shared experiences and highlight common issues.

- Raise the profile of the contribution of Black, Asian \& Minority Ethnic staff.

- Influence strategy and service development.

- Ensure all staff reach their potential.

What we did All staff from a Black, Asian \& Minority Ethnic background, in all services across the hospice, were personally invited to the first network meeting. It was held face-to-face, in a COVID-19 secure environment. The network was led by a member of the finance team and Chair of the Employee Engagement Group, with an academic fellow who gave a presentation about COVID-19 and the impact on Black, Asian \& Minority Ethnic communities and the importance of vaccination.

How it's going: To date four meetings have been held, with over $75 \%$ of our Black, Asian \& Minority Ethnic staff attending at least one meeting. The group have set their own agenda, have shared their experiences of working at St Gemma's and had another presentation by one of the trustees, who has set up a similar network in primary care in the city. Staff are enthusiastic about the network, are keen to share and influence the organisation, with the next steps of agreeing a name for the group, creating some terms of reference and a plan for the year.

\section{P-205 CULTURE ENGAGEMENT SURVEY}

Diane Jenner. Heart of Kent Hospice, Aylesford, UK

\subsection{6/spcare-2021-Hospice.220}

Background In 2019 the hospice launched our Culture Framework which gave us one shared common definition of our culture. Our purpose and values were translated into a mind-set and four behaviours. The annual colleague survey was developed to provide insight into how colleagues experience this culture in their work, enabling us to track whether the culture was being seen and felt in action. The survey aims to:

- Establish a snapshot of the culture framework in action.

- Assess levels of engagement, perspective on the current culture, inform strategy and identify priorities.

- Better understand enablers and barriers to completion.

- Track our journey to date.

Methods Using an online survey to gather quantitative and qualitative data, which focused on how colleagues experienced the four behaviours of 'I care', 'I learn', 'I own' and 'I improve' in action. The response rate was a tool to assess the level of engagement with the culture framework.

Results The results demonstrated a high level of engagement with the response rate improving from $62 \%$ in 2018 to $85 \%$ in 2020. The results provided insight into how colleagues experience their work, and measured how well we are living the defined purpose, values, mind-set and behaviours. It enabled us to understand how important the culture is for creating a great place to work.

Conclusions The survey is an important platform to listen, understand, improve, and encourage further dialogue. If we are fully engaged in our work, we can provide even better care to our patients, their families and the communities we serve. Colleagues believe in the importance of the culture framework and are committed to living it to provide an outstanding service.

\section{P-206 PROGRESSING THROUGH THE PATH OF THE PANDEMIC WITH DYSLEXIA - A SENIOR LEADER'S PERSPECTIVE}

Tracy Cunningham. St Luke's Hospice, London, UK

\subsection{6/spcare-2021-Hospice.221}

Introduction I am Assistant Director of Care and Quality at St. Luke's Hospice working internally and externally with health and social and voluntary care providers. I have dyslexia and was undergoing coaching and training on the use of new software for my dyslexia when the pandemic struck.

Aims To raise awareness of dyslexia and hidden disabilities, the specific challenges it has brought through the pandemic with implications for the wider workforce and the impact of not being able to continue my dyslexia support from an external organisation.

Discussion I was required to formulate a prompt response to the pandemic and rapidly changing guidance to inform, direct and support the diverse wider workforce. The reduction of face-to-face contact with colleagues, and a significant increase in demand for visiting dying patients at home presented huge challenges with the introduction of virtual technology. This impacted on my role both professionally and personally, requiring me to declare my dyslexia openly more than ever before.

In a position of influence, having strategic vision and role modelling remained crucial whilst promoting inclusiveness that empowered others. Emergency response meetings meant information could not be shared early, a key consideration for staff with dyslexia. Assimilation of the information to make this meaningful and implementation of new ways of working was a constant challenge whilst supporting others at a crucial time. The Equality Act and what this truly means in practice came into sharp focus. Training around facilitative technology for dyslexia was suspended replaced with selftaught techniques alongside the need to be continually creative and resilient.

COVID-19 provided an opportunity to raise awareness and highlight the specific needs that staff with dyslexia may have when required to work at pace within an ever changing landscape. It was imperative that the same compassionate tolerance, inclusivity and fairness, cornerstones of hospice care provision, was afforded to all staff at this time, particularly those with dyslexia.

\section{P-207 PEOPLE WANT TO HELP, BUT WILL WE LET THEM? VIEWS ON VOLUNTEER INVOLVEMENT IN END-OF-LIFE CARE}

1,2 ${ }^{3}$ Jessica Blake, 'Guy Peryer. 'University of East Anglia, Norwich, UK; ${ }^{2}$ James Paget University Hospital, Great Yarmouth, UK; ${ }^{3}$ East Coast Community Healthcare, Lowestoft, UK

\subsection{6/spcare-2021-Hospice.222}

Background The National Health Service Long Term Plan (2019) recommends that healthcare services work in partnership with the voluntary sector, and aims to double the number of healthcare volunteers by 2035. This includes volunteering in end-of-life care, a sector with a strong 
commitment to personalised care in a person's preferred place. During the COVID-19 pandemic, an appetite for healthcare volunteering was revealed.

Objective This exploratory study aimed to understand health and social care professionals' views on volunteer involvement in rapid transfers from hospital to a preferred place for a person nearing the end-of-life, and whether professionals perceived volunteers as being able to offer meaningful support.

Methods 13 semi-structured interviews were conducted between December 2019 and February 2020 with stakeholders working in health, social care, and the voluntary sector. An inductive thematic analysis of interview data was conducted.

Results 'Filling gaps', 'pastoral and practical support', 'a point of liaison', and 'robust volunteer management' were identified as themes. Participants reflected healthcare-led and risk-averse perspectives on volunteer involvement. Volunteers were perceived as being able fill gaps in service provision and to augment currently available pastoral, social and practical support. They were also seen as helpful go-betweens between patients and professionals. Participants stipulated that volunteer involvement in rapid transfers of care would require robust screening measures, boundaries, and support via a dedicated volunteer co-ordinator.

Conclusion Professionals expressed a paternalistic view of volunteer involvement, which is likely to impact the extent to which rapid transfer volunteers would be utilised, and their level of autonomy. The sustainability of volunteer involvement in this area is therefore of concern, as this approach is unlikely to benefit or value the volunteers involved. Before a recruitment drive takes place, generating greater confidence in volunteer ability and accountability via healthcare policy may be a possible solution. Further research exploring volunteer perspectives is required.

\section{Transformation}

\section{P-208 STRATEGIC PARTNERSHIP BETWEEN HOSPICES - THE OPPORTUNITIES AND BENEFITS OF HOSPICES WORKING COLLABORATIVELY}

${ }^{1}$ Kate Heaps, ${ }^{2}$ Heather Richardson, ${ }^{2}$ Shaun O'Leary. 'Greenwich and Bexley Community Hospice, London, UKi ${ }^{2}$ St Christopher's Hospice, London, UK

10.1136/spcare-2021-Hospice.223

Background With changes in NHS commissioning which aim for greater integration across larger areas and increased resourcing pressures experienced by hospices; strategic partnerships between hospices have never been more necessary. The recent Hospice UK Future Vision Programme (2020) set out principles of sustainability for hospices, many of which can be best achieved by working together.

In 2017 our two hospices established a partnership; managed through a memorandum of understanding (MOU), it set out a shared ambition, agreed principles for engagement and the partnership's governance structure.

Aims The partnership agreed to:

- Work together with integrity and probity for the benefit of patients and the public.

- Improve outcomes and experiences through early collaboration and planning.
- Deliver high-quality standards of patient care, consistently and equitably across the system.

- Lead the way in strategic planning of services that are safe and sustainable

- Identify/share opportunities for good practice in transparent and value-for-money partnership arrangements.

Achievements so far/Results Our hospices have developed supportive relationships and regular communication between leadership teams, operational managers and boards. Working collaboratively in:

- Joint external education delivery - sharing resources, contacts and income.

- Rehabilitation - supporting development of one partner's service through senior leadership and joint occupational therapy post.

- Fundraising - trialling a joint mass-participation event.

- Retail - supporting personal development of new income generation director and establishing improvements for retail.

- Commissioning -to support negotiations with commissioners and increase influence across integrated care system.

- Medical support - sharing senior medical resource to support development/business continuity.

- Bed capacity - sharing inpatient capacity during COVID-19 related closures.

- Staff wellbeing - shared health/wellbeing month funded by NHS England.

Conclusions By working together, we've achieved service improvements, shared expertise, increased efficiency and maintained business continuity. We continue to look for opportunities to work together for the furtherance of our stated aims as outlined in the MOU.

\section{P-209 STRENGTH IN NUMBERS - AN ICS-WIDE CEO ALLIANCE}

Sue McGraw. St John's Hospice North Lancashire and South Lakes, Lancaster, UK

10.1136/spcare-2021-Hospice.224

Hospice chief executive officers serving the Lancashire \& South Cumbria (L\&SC) ICS area began to meet weekly at noon each Thursday, initially, as a response to the pandemic. Throughout the year, as trust and partnership working grew, a number of successes and initiatives emerged. The main one being an excellent relationship within our newly developing ICS.

During the initial stages of the COVID-19 pandemic a marked increase in demand for community end-of-life care was experienced within L\&SC. After a presentation to the ICS by the 8 adult hospices, a discussion took place regarding the critical partnership role the hospice sector had played in enabling the system to respond to the changing preferences of the population during that period.

ICS Board members noted the potential opportunity this presented to increase joint working between the hospice sector and Out of Hospital services during the Winter period to support system resilience. After further discussion with the ICS, the opportunity was identified to undertake a rapid pilot scheme to demonstrate how a small amount of additional funding $(£ 200 \mathrm{k})$ provided at system level could increase the retention of vulnerable end-of-life patients in the community during the Winter period as part of the system COVID-19 management approach. 